\title{
QoS Framework for a Multi-stack based Heterogeneous Wireless Sensor Network
}

\author{
K. Panimozhi, G. Mahadevan \\ Department of Computer Science and Engineering, Visvesvaraya Technological University, India
}

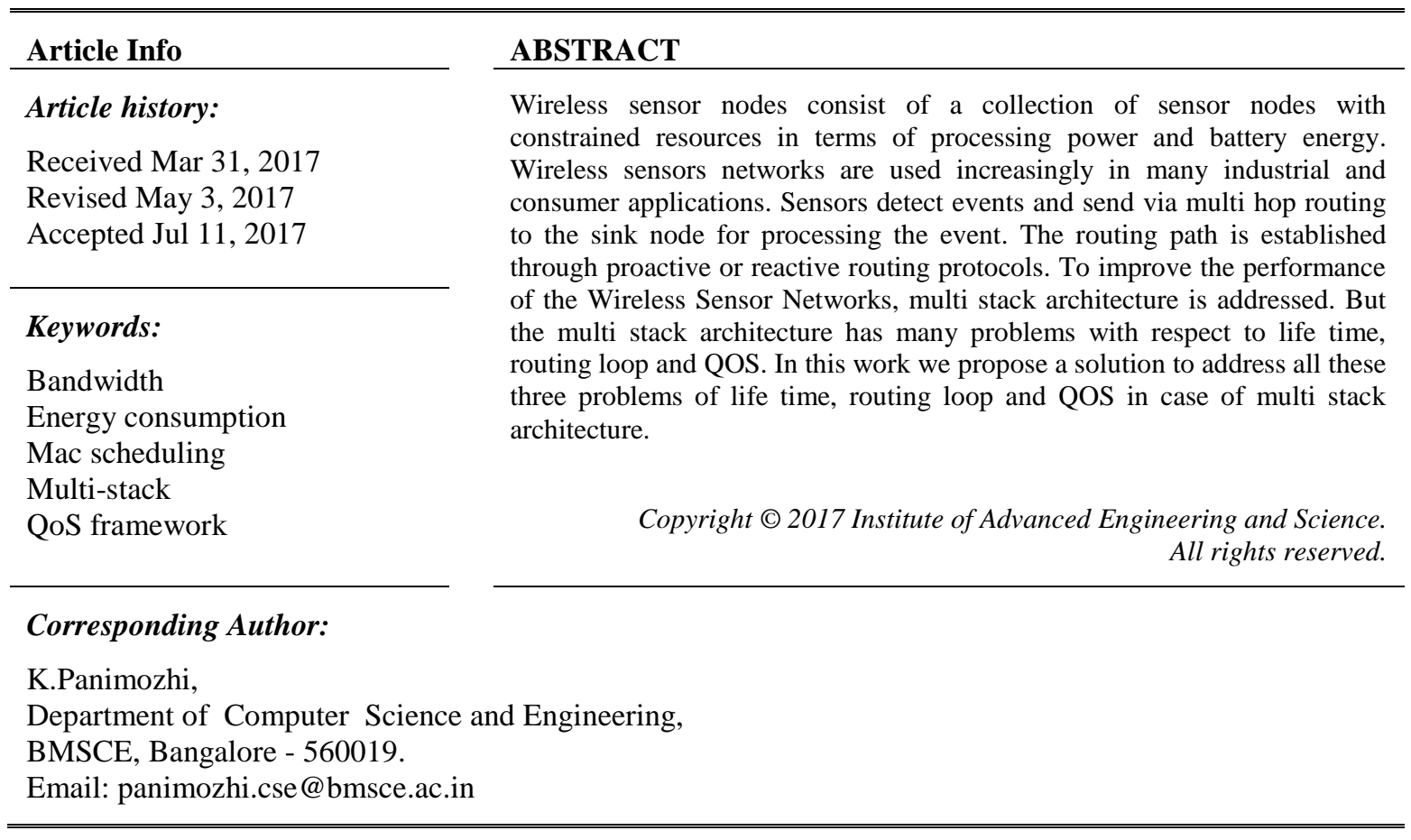

\section{INTRODUCTION}

Sensor networks have been playing a vital role with evolution of technologies for sophisticated use by mankind. Optimization of sensor networks on several parameters has made the utilization of these networks in several spheres of human life, for e.g. blooming of Internet of things. But to develop a sensor network that supports recent day's advancement in technology needs a unified framework to enable existence of different types of application on the same sensor network. Such kind of versatile sensor networks will have lots of limitations while designing. But when these limitations are overcome, they can be used for more than one application simultaneously.

This paper aims at providing a general framework which supports major Quality of service metrics that would benefit the end users to utilize their WSN based heterogeneous applications efficiently. Framework provides a unified structure enabling varied applications to run efficiently and effectively. In this regards few attempts have been made by us to design such kind of framework. And our study has been contributed in the paper [1], in which priority based packet scheduling algorithm was implemented on a multi-stack to increase the throughput and decrease delay for an application with mixed traffic of a wireless sensor networks. Next, dynamic bandwidth utilization algorithm was implemented on the same approach to efficiently use the bandwidth allocated for different schedules on the multi-stack queue.

\section{RELATED STUDY}

The sensor constituting the wireless sensor networks are capable of sensing and as well as communicating with other sensors within its spatial regions [2]. This capability has made the use of these sensors in wide variety of applications. But its constraints on low battery span, limited energy has refrained these types of networks from supporting multiple application or different types of data. 
Huge effort has been made by many researchers to overcome these kinds of constraints to support applications with varied data types. In [3], the authors have formulated an allocation scheme for channel to support various classes of QoS on the spectrum using Markovain models. This scheme has been tested with real world reading for a wireless medical sensor network and the results proved good. In [4], the issues such as less energy efficiency, loss of packet, longer jitters related to congestion have been overcome by proposing optimized energy consumption based congestion avoidance and detection mechanism.

In [5], several performance metrics such as fault, delay tolerance, reliability which contribute to overall performance of the wireless network sensor network has been considered to provide a framework that monitors the overall network performance. In [6], a general formula on the lifetime for WSNs that is distinct to any network system has been proposed. The proposed formula is based on a greedy method that ignores the channel state information and energy information which helps in improvement of network lifetime. In [7], the authors address the problem of reconfiguring and allocating the bandwidth using a frame work which uses probabilistic and component-based design principles. In this work the authors have analyzed the performance of network using admission control manager for both reliability and energy- conservation.

In [8] the authors have developed a utility frame work that is most suited for heterogeneous sensor networks, carrying inflexible traffic and to provide efficient rate control and fair resource allocation mechanism. This framework has been developed to support any sensor network which is energy constrained. In [9], [10], [11] and [12] data with different priority or data with varied importance is handled. These techniques differentiate data generated by different sensing tasks so that more sensitive data is given higher priority. The sensor networks with multiple rate can be handled effectively using weighted fairness is the suggestion given by authors of this paper. Wireless sensor networks paly a very important role in monitoring applications. In recent times the same network deployed is forced to handle multiple applications with varied QoS requirement. There are many cross layer approaches designed to handle these requirements. On such approach which is orthogonal to it is Multi-stack approach in which different pairs of MAC protocols and network protocols are handled [13].

Recently researchers are contributing more on the power conservation or energy efficiency in Wireless Sensor Networks which is a prime issue in deciding the longevity of these networks. In [14], a review on various ways of managing the energy consumption inorder to increase the life span of the network is discussed. The authors have provided a theoritcal model to improve the network life time by modifiying the existing routing algorithms. In [15] the authors have given a new approach to improve the energy efficiency in hetergeonus network using a fuzzy based alogirthm in combination with neural networks.

\section{RESEARCH METHODOLOGY}

\subsection{PROBLEM DEFINITION}

Given a wireless sensor network covering an area of A and $\mathrm{N}$ sensor nodes distributed randomly in the network with a single sink. Each sensor Node N has M stack and charged with battery capacity B. Sensor detects and report events to Sink. The data from sensors can be scheduled on any stack and due to operation of all stacks, the node's energy will drain at faster rate. Due to multiple stacks, the data routed from node may be looped to same node and not delivered to sink. Some Nodes in the network may be too selfish and want to use the entire bandwidth and in this case, other nodes are robbed their share of bandwidth. In this paper we deal with these three problems and propose solution for the same:

a. Increase the life of network in the multi stack sensor networks

b. Manage the bandwidth and ensure fair usage

c. Avoid routing loops in the network.

\subsection{PROPOSED SOLUTION}

In order to popularise the use of wireless sensor networks to support for multiple independent applications it is necessary to provide a base or infrastructure, such that different users can concurrently submit their applications to accomplish diverse goals. The proposed solution has two parts:

a. Initialization Stage

b. Routing Stage

\subsubsection{INITIALIZATION STAGE}

In the initialization stage, the sensor network is organized into clusters. The nodes which cover the same sensing area are grouped into clusters. For each cluster, a cluster id is chosen and informed to all the nodes in the cluster. Any node in the cluster can process the packet addressed to its cluster. In the cluster, only one node is active at time which is decided by round robin among the nodes in the cluster. 
The cluster formation algorithm:

a. Each Node broadcasts a hello packet for 1 hop and wait for some threshold time

b. Every node receiving the hello packet will keep the information of neighbor from where hello packet is received

c. After the threshold time, node broadcasts its neighbor information received.

d. A node which has more number of neighbors becomes the cluster head and all the nodes join in that cluster.

e. The cluster head node creates duty cycle time schedule for the nodes in that cluster and also creates a unique cluster id. Unique cluster id among network can be created by doing a mathematical operation on its cluster node id. [Say id $* 10000=$ Cluster id]

f. Cluster head sends instruction to sleep, for all cluster nodes and wait for Hello route packet from the sink node.

g. Once the shortest route to sink is built at the cluster head, it instructs all cluster nodes to wakeup.

h. Cluster head sends the duty cycle and the cluster id to all nodes within the cluster and start the duty cycle phase.

Once the clusters are created, the sink will start the route building process. Sink broadcasts Hello packet and 1 hop neighbor nodes receiving it, will append its cluster id and rebroadcast the Hello packet. Also node remembers the short hop to the sink node by viewing the path in the Hello Packet. This makes every cluster aware of, through which cluster the packet has to be routed with less hop to the Sink. The route building process:

a. Sink broadcasts a hello packet with hop count as 0 and its id.

b. One hop neighbor Node receiving it, will update its route list with path to sink, append its cluster id and rebroadcast to its neighbors.

c. Each node will update, in this way a shortest hop to sink will be recorded.

By this Initialization stage, two of the issues in the problem statement is solved. Since clustering is done and only one node in cluster is awake at a time, energy saving is made without affecting the sensing accuracy. Also routing loop is avoided in this approach, as the routing path is trigged from Sink and each node stores the shortest path to sink as the route.

\subsubsection{ROTUING STAGE}

Routing stage starts for every time, a sensor node senses data. Routing needs bandwidth for packet transmission. One of the main reason for QOS distortion is due to the fact that bandwidth is not managed in the network. This issue is overcome by our approach as we manage the bandwidth though routing to obtain better QOS in the network. The routing steps in the proposed solution is as follows :

1. Each node before starting a session with sink must send a B_RESERVE information in the first packet to claim the data rate needed. This is needed for bandwidth reservation.

2. Once the intermediate node receives B_RESERVE information it will try to allocate a bandwidth on any one of stack according to following rules an in Figure 6.

Rule 1: Bandwidth needed is split to three ranges Low / Medium/ High

Rule 2: If the bandwidth asked in B_RESERVE is Low, then route from low to high shortest hop count is searched in routing list for allocation.

Rule 3: If the bandwidth asked in B_RESERVE is medium, then route from middle value to high shortest hop count is searched in routing list for allocation

Rule 4: If the bandwidth asked in B_RESERVE is high, then route from high to low shortest hop is searched in routing list for allocation

Rule 5: The route is selected if the requested bandwidth can be allocated on a stack for that route and once route is selected, the maximum bandwidth is reduced by the amount allocated

3. After route is selected, it is filled in the packet and forwarded to next hop

4. Source node must send B_RESERVE information in the packet after an interval of d, else at interval of $d$, intermediate nodes will release the bandwidth allocated

5. To discourage nodes from abusive use and ensure fair usage of bandwidth, the bandwidth allocation on subsequent B_RESERVE is controlled as

$\mathrm{Bw}$ to allot $=\mathrm{BW}$ asked in B_RESERVE - numtimes B_RESERVE $*$ step, where step is a configured value.

6. Also to reduce QOS for more aggressive users of bandwidth, bandwidth is allocated on longest path even when the B_RESERVE value is low. To control this allocation of bandwidth based on B_RESERVE value, number of times requested, QOS feedback a fuzzy logic based selection will be used 
7. Each node collects QOS feedback on all stacks to measure the current QOS in terms of packet loss and based on it decide to allocate new request for bandwidth.

To control the allocation of bandwidth, a fuzzy logic decision is made as in Figure 1. The decision is based on Requested reserve value, number of times requested within last $\mathrm{N}$ duration, QOS feedback in the last $\mathrm{N}$ duration.

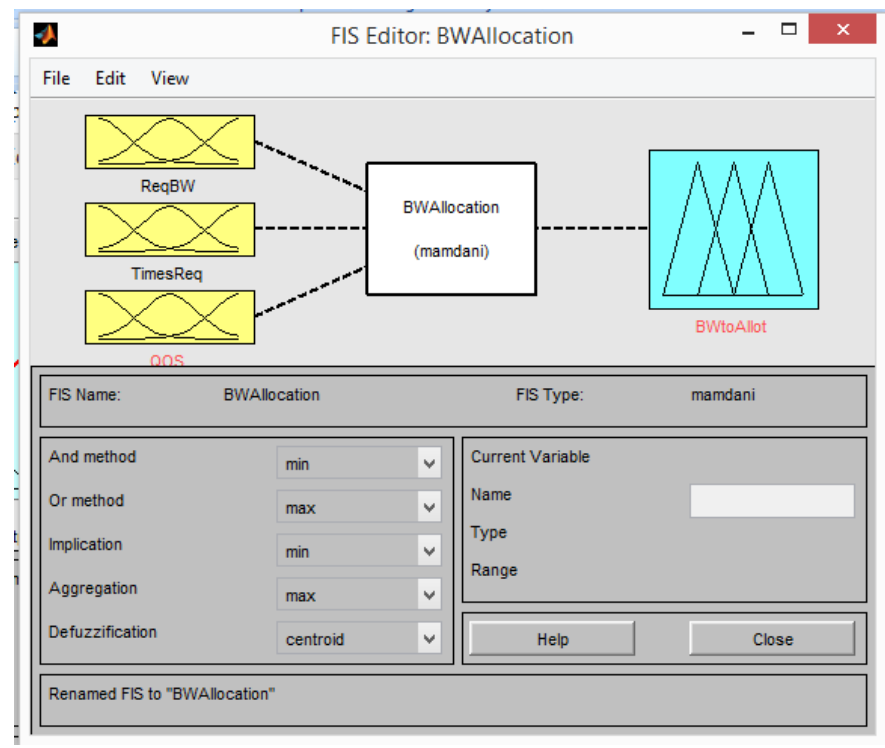

Figure 1. Fuzzy based decision for bandwidth allocation

The Requested reserve value is split into three categories L, M, H normalized by the maximum value that can be requested. The number of times requested is split into three categories $\mathrm{L}, \mathrm{M}, \mathrm{H}$ normalized by the maximum number of times of request within duration $\mathrm{N}$.

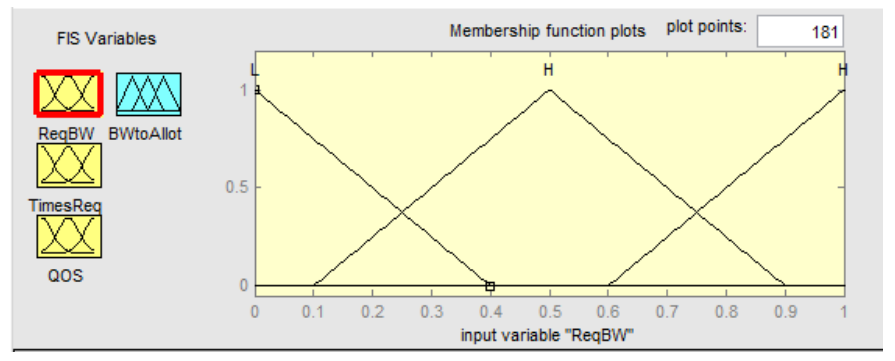

Figure 2. The membership functions for ReqBW

Step 2 to step 4 are derived using the membership function to assign the requested bandwidth through the Figure 2. Step 5 which is used to avoid the repeated request by a specific node is identified and avoided by the membership function for TimesReq. TimesReq denotes number of times the bandwidth is requested by a specific node. QOS feedback is measured in terms of these inputs, the bandwidth to allocate is given as output variable. The final output of allocating the bandwidth at step 5 is represented in the Figure 5. The allocation happens after analyzing the level of bandwidth requested and number of times the bandwidth is requested. 


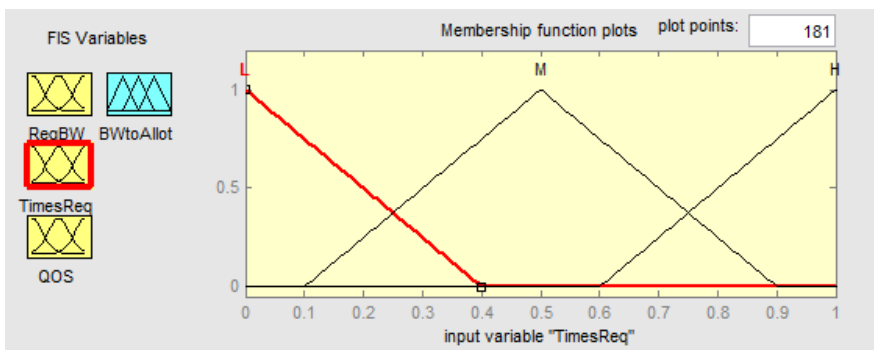

Figure 3. The membership functions for TimesReq

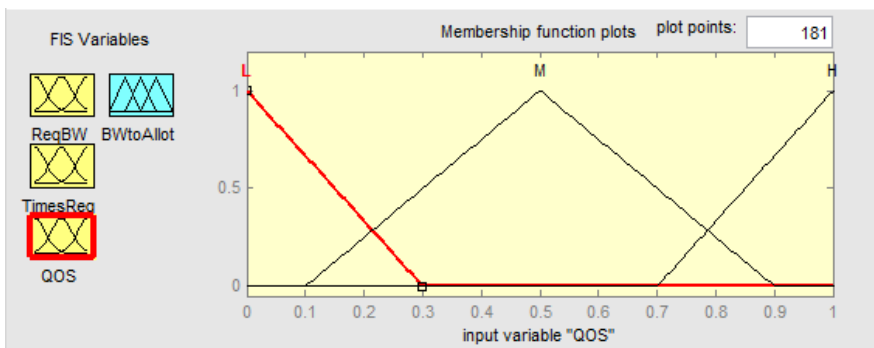

Figure 4. The membership functions for QOS Feedback

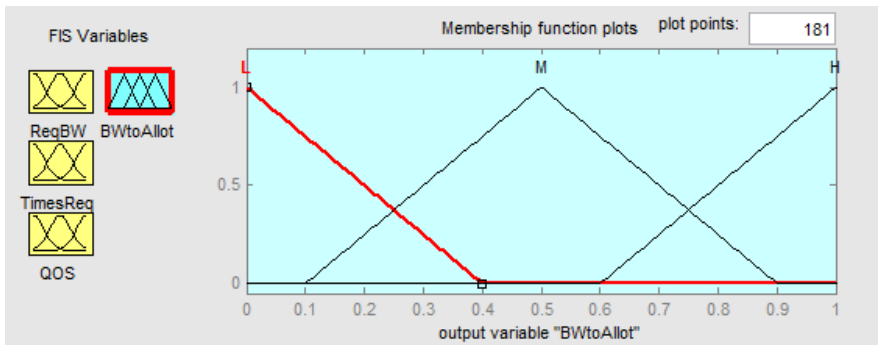

Figure 5. The membership functions for the output variable bandwidth to allocate

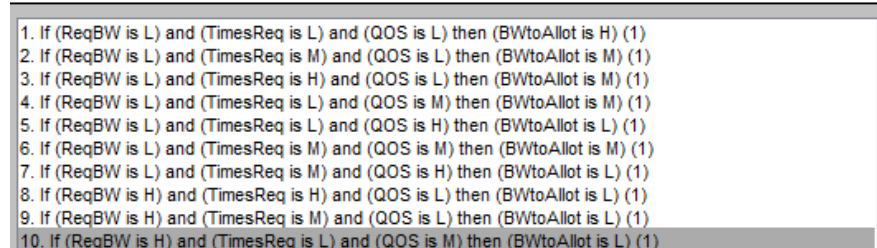

Figure 6. The rules for input to output translation

\section{RESULTS}

To test the performance of proposed method we implemented the solution using JProwler. The simulation was conducted with the parameters shown in Table 1. The simulation was carried comparing the proposed solution (Multi stack with bandwidth management), multi stack and single stack solution. Following four parameters are collected and measured :
a. Throughput
b. Delay
c. Packet Loss
d. Average Energy consumption
e. Life time 
Table 1. Simulation Parameters

\begin{tabular}{ll}
\hline Parameters & Values \\
\hline Number of Nodes & Upto 100 Nodes \\
Communication range & $100 \mathrm{~m}$ \\
Area of simulation & $800 \mathrm{~m}^{*} 800 \mathrm{~m}$ \\
Packet Rate & 10 to 25 packet per second \\
Simulation time & 100 seconds \\
Interface Queue Length & 50 \\
MAC & SMAC/TMAC \\
No of sink & 1 \\
No of Stack & 2 \\
\hline
\end{tabular}

Throughput is measured in terms of number of packets received at sink.

Throughput vs Network Density

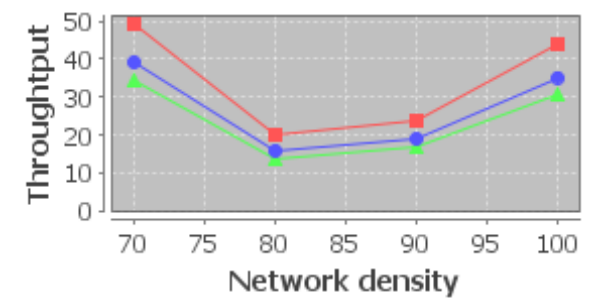

--Multistack with bw,energy mgmt $-\bullet-$ Multistack - Single Stack

Figure 7. Throughput vs Network density
Throughput vs Data rate

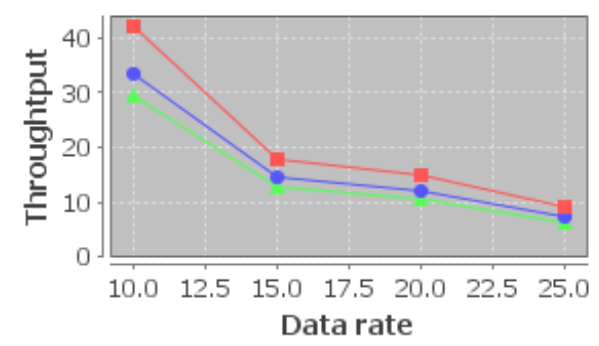

- - Multistack with bw,energy mgmt - - Multistack

- Single Stack

Figure 8. Throughput vs Data rate

The network density is varied and the throughput is measured and plotted. From the Figure 7, we see that throughput is high in the proposed multi stack with BW-energy management compared to other solutions. Throughput is compared for varying data rate, and from the Figure 8, we see that the throughput is higher in proposed solution compared to others. Delay is measured in terms of average latency of packets from source to sink.

\section{Delay vs Network Density}

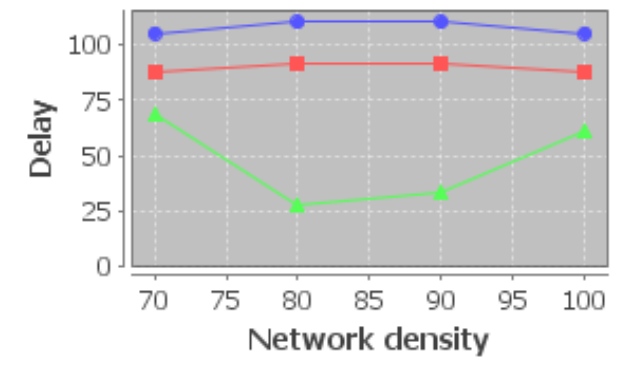

- Multistack with bw,energy mgmt - $\bullet$-Multistack

- Single Stack

Figure 9. Delay Vs Network Density

\section{Delay vs Data rate}

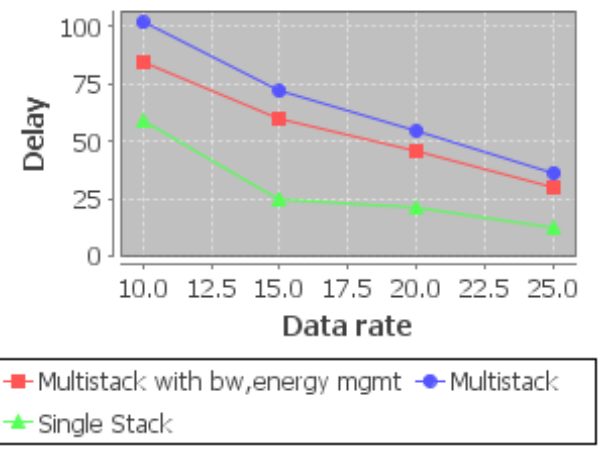

Figure 10. Delay vs Data rate

Delay is measured by varying the number of nodes and data rate, and from the Figure 9 we see that the proposed solution has slightly higher delay because of bandwidth restriction. For different data rates, the 
delay is measured. From the Figure 10, we see that the delay is slightly higher in proposed because of bandwidth restriction. Packet Loss is measured in terms of packet loss ratio.

\section{Packet Loss vs Network Density}

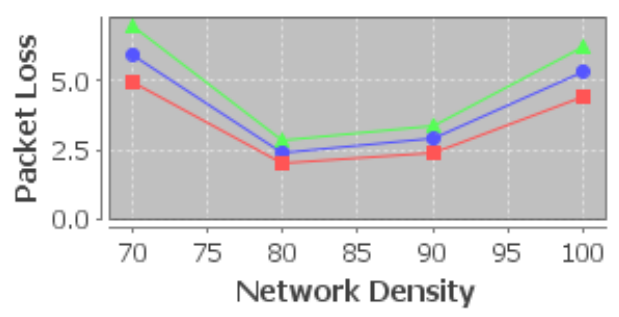

-m-Multistack with bw,energy mgmt - - Multistack - Single Stack

Figure 11. Packet Loss vs Network Density
Packet Loss vs Data rate

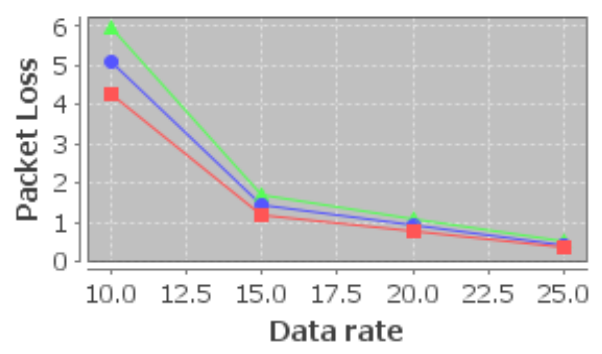

- Multistack with bw,energy mgmt - - Multistack

- Single Stack

Figure 12. Packet Loss vs Data rate

Packet loss is measured by varying the number of nodes in the network. From the Figure 11, we see that the packet loss is less in proposed method compared to the other solution.Packet loss is measured by varying the data rate and from the Figure 12, we see that packet loss is slightly lower in the proposed compared to other solutions.

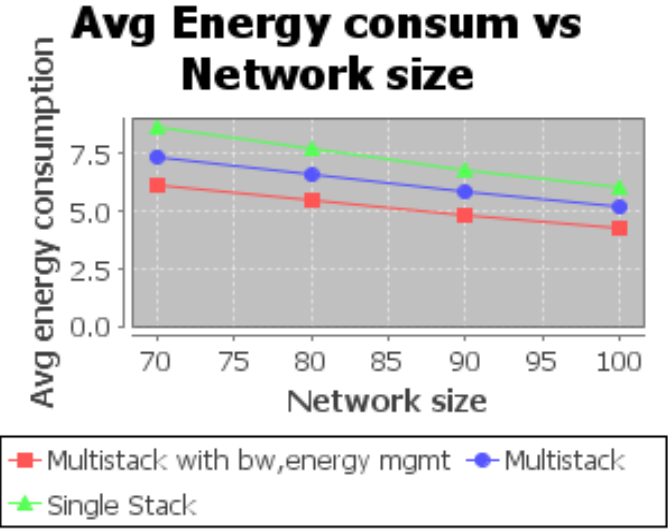

Figure 13. Average Energy consumption vs Network Size

\section{Life time vs Network size}

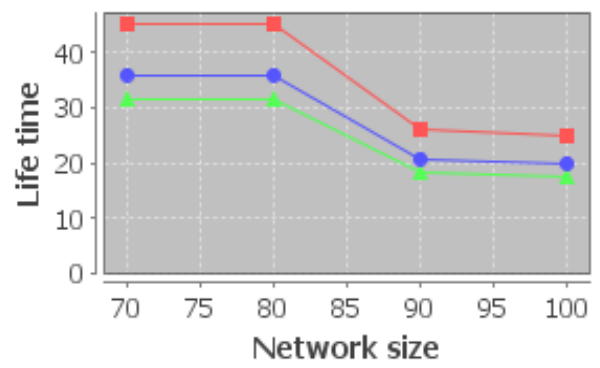

- - Multistack with bw,energy mgmt - $\bullet$-Multistack

- Single Stack

Figure 14. Lifetime vs Network size

The average energy consumption is measured by summing the residual energy at all nodes and dividing it by number of nodes. From the Figure 13, we see that in proposed solution average energy consumption is less because of the clustering based routing used in the proposed solution. The life time of the network is measured in terms of time at which the first node residual energy becomes 0 , and from the Figure 14, the proposed solution has more life time compared to other solution proposed by us.

\section{CONCLUSION}

In this work, multiplicity of factors has been taken into consideration when designing a QoS framework for a Wireless Sensor Network. These factors may also change during the lifetime of the network based on type of packet, energy constraint etc,. But, in this work, major parameters such as bandwidth utilization, routing loop occurrence etc., that would greatly affect the network life time has been considered to improve upon the network lifespan. As a future work, we have planned to design Zigbee based WSN to test the algorithm designed so as to facilitate the use of this framework in real-life scenarios. 


\section{REFERENCES}

[1] Panimozhi K and G Mahadevan.,"Multi-Stack Architecture Implementation to Enhance the QoS in WSN with Prioritization of Packets", International Journal of Computer Applications 120(23):51-56, June 2015.

[2] F. Akyildiz, W. Su, Y. Sankarasubramaniam, and E. Cayirci, "Wireless sensor networks : a survey," Comput. networks, vol. 38, pp. 393-422, 2002.

[3] Rahman Doost-Mohammady, M. Yousof Naderi, and Kaushik Roy Chowdhury," Spectrum Allocation and QoS Provisioning Framework for Cognitive Radio With Heterogeneous Service Classes, "IEEE Transactions on Wireless Communications, Vol. 13, No. 7, JULY 2014.

[4] Liqiang Tao, Fengqi Yu, "A Novel Congestion Detection and Avoidance Algorithm for Multiple Class of Traffic in Sensor Network", Proceedings of the 2011 IEEE International Conference on Cyber Technology in Automation, Control, and Intelligent Systems March 20-23, 2011, Kunming, China

[5] Vasco Pereira, Jorge Sá Silva, Edmundo Monteiro, “A framework for Wireless Sensor Networks performance monitoring", IEEE 2012.

[6] Yunxia Chen and Qing Zhao," On the Lifetime of Wireless Sensor Networks", IEEE Communications Letters, Vol. 9, No. 11, November 2005.

[7] VW Mahyastuty, AA Pramudita, "Low energy adaptive clustering hierarchy routing protocol for wireless sensor network," TELKOMNIKA Telecommunication Computing Electronics and Control., vol. 12, no. 4, pp. 963-968, 2014.

[8] Jiong Jin,Marimuthu Palaniswami, Bhaskar Krishnamachari, Rate control for heterogeneous wireless sensor networks: Characterization, algorithms and performance,Elsevier, September 2012.

[9] C. Wang, B. Li, K. Sohraby, M. Daneshmand and Y. Hu, "Upstream Congestion Control in Wireless Sensor Networks through Cross-layer Optimization," IEEE Journal on Selected Areas in Communications, Vol. 25, Issue. 4, May 2007, pp. 786-795.

[10] C. Wang, K. Sohraby, V. Lawrence, B. Li and Y. Hu, "Priority-based Congestion Control in Wireless Sensor Networks," IEEE International Conference on Sensor Networks, Ubiquitous and Trustworthy Computing 2006, Vol. 1, June 2007, pp. 22-31.

[11] I.C. Paschalidis, W. Lai and D. Starobinski, “Asymptotically Optimal Transmission Policies for Large- Scale LowPower Wireless Sensor Networks," IEEE/ACM Transactions on Networking, Vol. 15, Issue. 1, Feb 2007, pp. 105118.

[12] I.C. Paschalidis, W. Lai and D. Starobinski, "Asymptotically Optimal Transmission Policies for Low- Power Wireless Sensor Networks," IEEE Proceedings on the 24th Annual Joint Conference of the IEEE Computer and Communications Societies INFOCOM 2005, Vol. 4, Mar 2005, pp. 2458-2469.

[13] Nancy El Rachkidy, A. Guitton ,and M.Mission, "Improving QoS in wireless sensor networks using a multi-Stack architecture", in IEEE Vechicular Technology Conference, 2011.

[14] Rajiv R Bhandari, K Rajasekhar," Study on Improving the Network Life Time Maximazation for Wireless Sensor Network using Cross Layer Approach", International Journal of Electrical and Computer Engineering (IJECE), Vol. 6, No. 6, December 2016, pp. 3080-3086.

[15] Amit Kumar, Kaushik, "A Hybrid Approach of Fuzzy C-means Clustering and Neural network to make Energy Efficient heterogeneous Wireless Sensor network", in International Journal of Electircal and Computer Engineering, Vol 6, No 2: April 2016. 